\title{
Control basado en PLC y monitoreo mediante HMI de la temperatura en el proceso de maceración y cocción en la elaboración de cerveza artesanal
}

\author{
PLC-based control and HMI monitoring of the temperature in the maceration and cooking \\ process in the craft beer production
}

\author{
E. Flores-García ${ }^{a}$, J. C. Quezada-Quezada ${ }^{b}$, R. V. H. Calderón-Medina ${ }^{c}$, D. Guardado- \\ Montes de Oca ${ }^{d}$
}

\begin{abstract}
:
The brewmaster, in charge of the craft beer production, for the fact itself of being craft made, has to deal with disturbances within the manufacturing process which tend to affect the product's quality as well as modify its taste; such disturbances occur in the wort maceration and cooking stages, where temperatures must be constant during these phases. As a concrete solution alternative proposal to this problem, without modifying the way in which the craft beer is made according to that established by UNESCO, in this work a cascade PID type control is used in order to accomplish control over a gas valve to regulate a fire flame generating the temperature inside a reactor in the maceration and cooking stages, in addition to implement a HMI for process monitoring. The present work constitutes a terminal academic project as implementation, focused on industry process automation.
\end{abstract}

Keywords:

Craft beer, HMI, Process automation, PLC, Temperature control

\section{Resumen:}

El maestro cervecero, encargado de la producción de cerveza artesanal, por el hecho mismo de ser artesanal, tienen que lidiar con perturbaciones dentro del proceso de elaboración que llegan a afectan la calidad del producto y modificar su sabor; tales perturbaciones se presentan en las etapas de maceración y cocción del mosto, dónde las temperaturas durante estas fases deben ser constantes. Como propuesta de alternativa de solución concreta a este problema, sin modificar la manera en que se elabora la cerveza artesanal de acuerdo con lo establecido por la UNESCO, en este trabajo se hace uso de un control de tipo PID en cascad a el cual efectúa el control de una válvula de gas para regular el fuego que genera la temperatura dentro del reactor en las etapas de maceración y cocción, además de implementar una HMI para el monitoreo del proceso. El presente trabajo constituye un proyecto académico terminal de implementación, con enfoque a la automatización de procesos en la industria.

\section{Palabras Clave:}

Automatización de procesos, Cerveza artesanal, Control de temperatura, HMI, PLC

\section{Introducción}

En términos generales, la producción artesanal elabora productos mediante la transformación de materias primas naturales básicas, a través de procesos de producción no industrial que involucran máquinas y herramientas simples con predominio del trabajo físico y mental. La preparación de cerveza también se realiza de manera artesanal, e implica el entendimiento de una serie de reacciones enzimáticas, microbiológicas, así como el control de determinados parámetros; lo que hace en este caso que el artesano se convierta en un estudioso y

\footnotetext{
a Autor de correspondencia, Universidad Autónoma del Estado de Hidalgo, Escuela Superior de Tizayuca, https://orcid.org/0000-00024606-8217, email: efloresg@uaeh.edu.mx

bUniversidad Autónoma del Estado de Hidalgo, https://orcid.org/0000-0003-3125-3708, email: jcarlos@uaeh.edu.mx

c Universidad Autónoma del Estado de Hidalgo, https://orcid.org/0000-0002-7292-6051, email: rafael_calderon @uaeh.edu.mx

d Universidad Autónoma del Estado de Hidalgo, https://orcid.org/0000-0001-7602-0241, email: gu261023@uaeh.edu.mx
} 
conocedor del proceso, por lo que debe cuidar detalladamente la ejecución de los procedimientos, de manera que se realicen apropiadamente [1], ya que de lo contrario, se podría obtener una cerveza de baja calidad o con características indeseadas. Para la elaboración de cerveza, los principales parámetros a tener en cuenta son la temperatura y los tiempos, sin dejar de mencionar que en ello también se deben realizar mediciones de otras variables en el transcurso del proceso, por ejemplo $\mathrm{pH}$ y densidad, entre otras. El bueno manejo de la temperatura en la elaboración de cerveza, en general, mejora la presencia de componentes volátiles y aromáticos, mejorando el sabor de la cerveza [2], lo cual evidentemente es parte de los aspectos clave, y consecuentemente uno de los secretos, en este tipo de procesos.

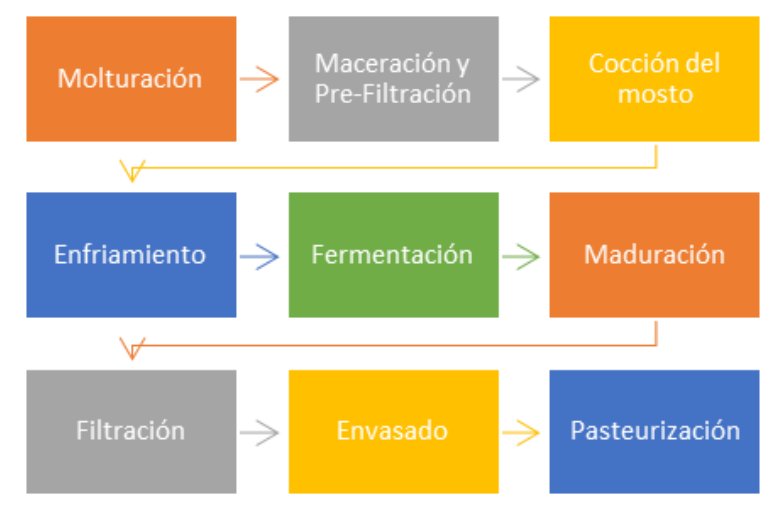

Figura 1. Diagrama de flujo del proceso de elaboración de cerveza artesanal.

\section{Automatización de procesos en la elaboración de cerveza artesanal}

\section{Análisis de problemática}

Con el propósito de realizar un análisis efectivo y proponer una alternativa de solución a la problemática presentada en el proceso de elaboración de cerveza artesanal, es necesario conocer al detalle las acciones y los eventos involucrados en los subprocesos de maceración y cocción, desde el ingreso de la materia prima al sistema, hasta los tiempos y las temperaturas de las diferentes etapas de elaboración de la cerveza [3].

En el diagrama de flujo de la Figura 2 se puede apreciar cada una de las acciones y los eventos que ocurren en las etapas de maceración y cocción en la producción de cerveza artesanal.

En gran medida, en los procesos de producción se ha presentado el control automático de sistemas como un aporte significativo de aplicación de tecnología para resolver satisfactoriamente las formas de supervisión, regulación, tratamiento y control de las tareas involucradas en los procesos. Para comprender de manera precisa lo que es la au tomatización, es necesario conocer como concepto general que un sistema de control automático es una interconexión de componentes que forman un arreglo definido, cuya configuración tiene la capacidad de comandarse por sí misma.

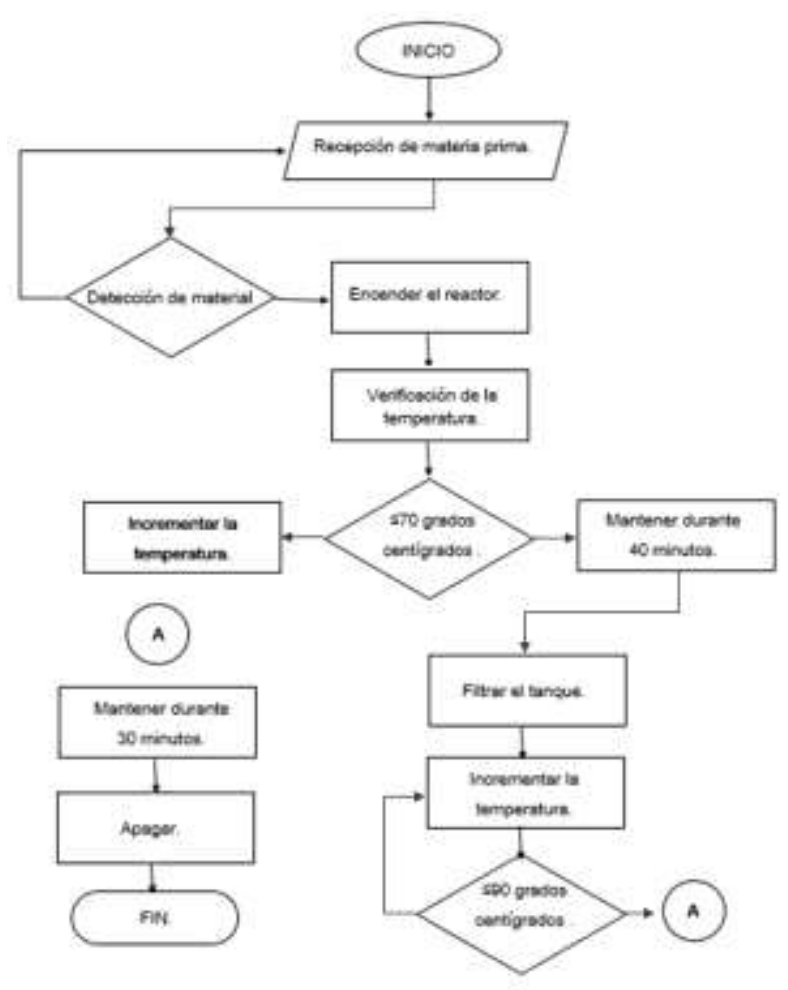

Figura 2. Diagrama de flujo de los procesos de maceración y cocción.

\section{Sistemas de control}

La Figura 3 muestra el diagrama básico de un sistema de control; la señal $r(t)$ representa la entrada al sistema o a un componente del sistema a ser controlado, con el objetivo de producirle una respuesta o salida $y(t)$ [4].

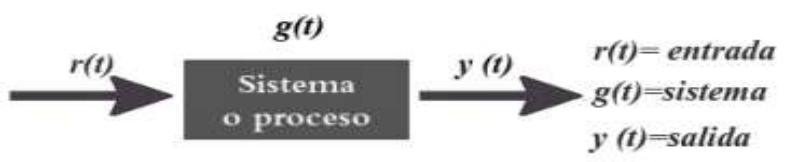

Figura 3. Elementos básicos de un sistema control.

\section{Módulos generales de control en lazo cerrado}

El controlador opera automáticamente produciendo un cambio en el valor deseado; se obtiene información del comportamiento dinámico del sistema para identificar un modelo de orden reducido para el proceso, o de las características de la oscilación sostenida del mismo, para u tilizarla en el cálculo de los parámetros del controlador. Un sistema automatizado de control en lazo cerrado se puede dividir en tres módulos generales, como se observa en la Figura 4. 
El módulo de supervisión, el de control, y el módulo operativo, cada uno de los cuales cuenta con sus elementos característicos que cumplen las respectivas funciones dentro de los mismos.

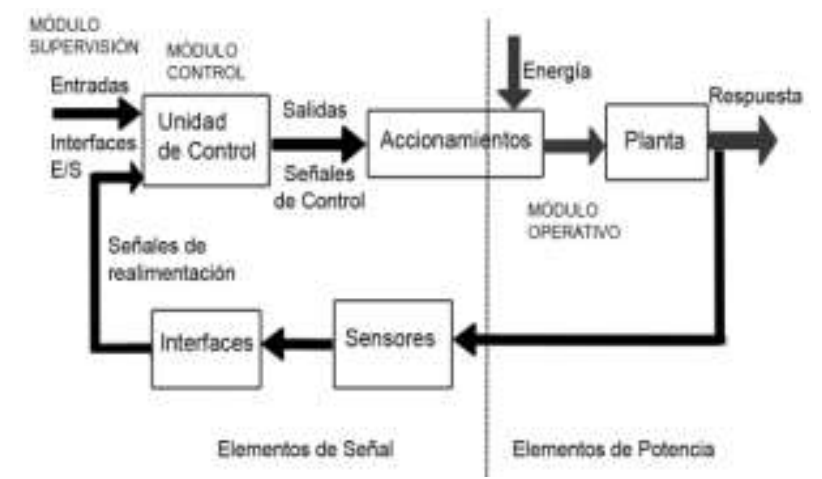

Figura 4. Módulos generales de un sistema de control en lazo cerrado.

\section{Control PID en cascada}

Uno de los métodos más utilizados para reducir considerablemente perturbaciones que entran en un proceso lento, es el control en cascada o circuitos múltiples. El control en cascada tiene también la capacidad de acelerar la respuesta del sistema de control, reduciendo la constante de tiempo de la fu n ción de transferencia del proceso que relaciona la variable manipulada con la salida de éste.

El control en cascada se conceptualiza como la configuración donde la salida de un controlador con retroalimentación es el punto de ajuste (set point) para por lo menos otro controlador con retroalimentación. En otras palabras, el control en cascada involucra sistemas de control con retroalimentación o circuitos que estén ordenados uno dentro de otro.

La Figura 5 muestra un diagrama de bloques que representa al sistema de control en cascada del proyecto desarrollado. Se puede observar que en lugar de aju star el elemento de control final, se ajusta en este caso la válvula reguladora; la salida del controlador primario es el punto de ajuste del lazo de control secundario [5].

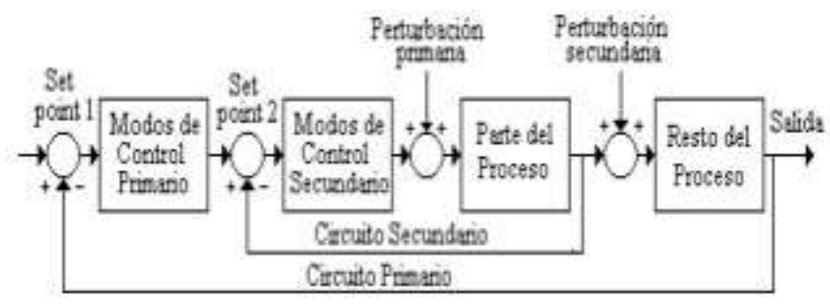

Figura 5. Diagrama en bloques de controladores conectados en cascada.
El circuito o lazo de control secundario que abarca sólo una parte del proceso total, es un sistema de orden menor o reducido, de manera que el controlador se puede ajustar para dar una respuesta más rápida; en el proyecto abordado se ha conectado en cascada un controlador de flujo con un controlador de temperatura.

Los controladores PID considerados por los autores de los métodos de sintonización, tienen algunas de las siguientes funciones de transferencia [6].

- Controlador PID-Ideal

$$
u(s)=K_{c}\left[1+\frac{1}{T_{i} s}+\frac{T_{d} s}{1+\tau_{f} s}\right] r(s)-y(s)
$$

- Controlador PID-Serie (Interactuante)

$$
u(s)=\dot{K}_{c}\left[1+\frac{1}{T_{\imath}^{\prime} s}\right]\left[\frac{T_{d}^{\prime} s}{1+\tau_{f} s}\right](r(s)-y(s))
$$

- Controlador PID-Industrial

$$
u(s)=K_{c}\left[1+\frac{1}{T_{l}^{\prime} s}\right]\left[r(s) \frac{T_{d}^{\prime} s}{1+\tau_{f} s} y(s)\right]
$$

Si el controlador PID que se desea sintonizar no es del mismo tipo que el supuesto en el método de sintonización a emplear, entonces se debe realizar la conversión de parámetros necesaria [5].

\section{Método de Ziegler-Nichols}

El primer procedimiento sistematizado para el cálculo de los parámetros de un controlador PID para su sintonización, fue desarrollado por Ziegler y Nichols [6]; de ahí que el método lleve sus nombres.

El criterio de desempeño que seleccionaron fue el de un decaimiento de $1 / 4$, es decir que el error decae en la cuarta parte de un periodo de oscilación. Las ecuaciones fueron determinadas de forma empírica a partir de pruebas realizadas en laboratorio con diferentes procesos, y están basadas en un modelo de primer orden más tiempo muerto identificado por el método de la tangente, para un funcionamiento del lazo de control como regulador con un controlador PID-Ideal.

En cuanto al tipo de controlador, si bien la gran mayoría de los autores advierten que este método es para la sintonización de controladores ideales, otros, entre los que figura Corripio [7], afirman que el método es para controladores que interactúan.

Las ecuaciones de sintonización de este método son: 


$$
\begin{gathered}
K c=1.2 \frac{\tau}{k p^{t} m} \quad \text { a } 2.0 \frac{\tau}{k p^{t} m} \\
T i=2 t_{m} \\
T d=0.5 t_{m}
\end{gathered}
$$

\section{Codificador (Encoder)}

El encoder, o codificador, es un transductor rotativo que, mediante una señal eléctrica, sirve para indicar la posición angular de un eje, velocidad y aceleración del rotor de un motor.

Un encoder está compuesto básicamente de un disco conectado a un eje giratorio. El disco comúnmente está hecho de vidrio o plástico y se encuentra "codificado" con unas partes transparentes y otras opacas que bloquean el paso de un haz de luz emitida por una fuente de luz, típicamente emisores infrarrojos. En la mayoría de los casos, estas áreas codificadas están arregladas en forma radial en el disco. Ver Figura 6.

A medida que el eje rota, el emisor infrarrojo emite la luz que es recibida por un sensor óptico o foto-transistor, generando pulsos digitales a medida que la luz cruza a través del disco o es bloqueada en diferentes secciones de éste. Esto produce una secuencia que es utilizada para controlar el ángulo de giro, la dirección del movimiento e incluso la velocidad [8].

\section{Encoder incremental}

Como su nombre indica, es un encoderque determina el ángulo de posición a través de la realización de cuentas incrementales.

Esto significa que el encoder incremental provee una posición estratégica desde donde siempre comenzará la cuenta. La posición actual del encoder es incremental cuando es comparada con la última posición registrada por el sensor. Los encoders incrementales son un tipo de encoder óptico, en los cuales cada posición es completamente única [8].

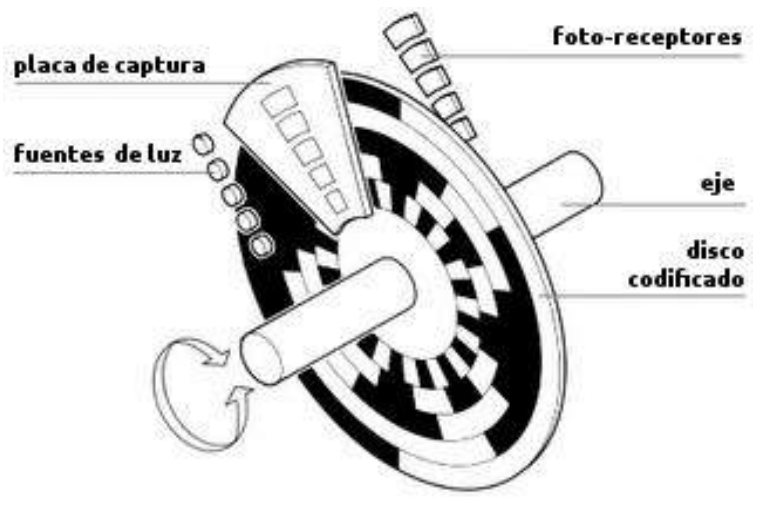

Figura 6. Encoderincremental.

\section{Algoritmo de Control}

Está conformado por un controlador PID en cascada, mostrado en los diagramas de la Figura 7 y Figura 8, con la finalidad de reducir lo más posible las pertu rbaciones que se pudieran presentar durante el proceso de elaboración de la cerveza artesanal, además de poder acelerar la respuesta del sistema de control reduciendo la constante de tiempo como se explicó anteriormente.

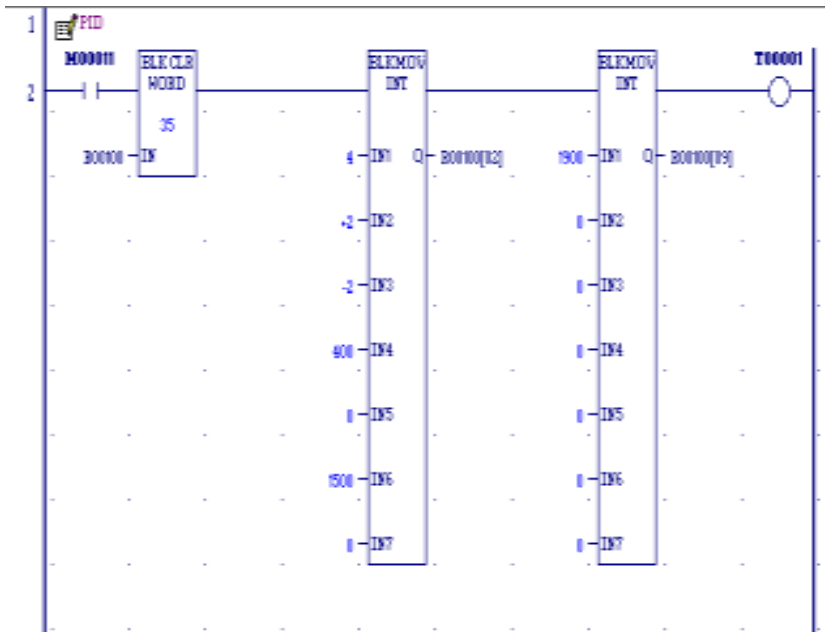

Figura 7. Algoritmo de control: Control PID en cascada.

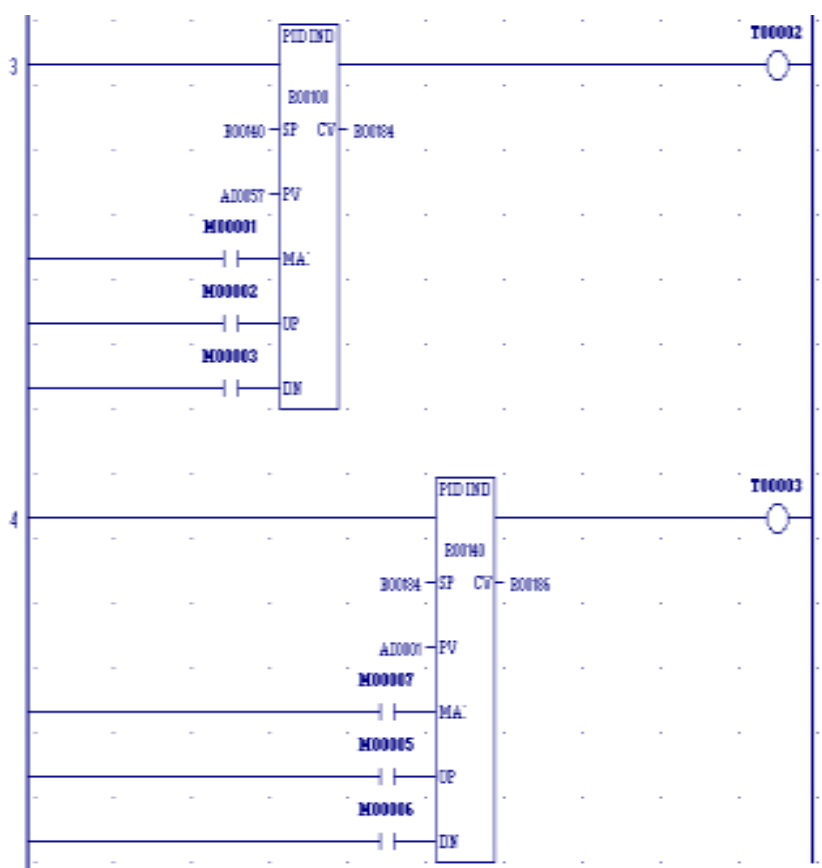

Figura 8. Algoritmo de control: Control PID en cascada.

En la Figura 9 y Figura 10 se presentan los diagramas del control efectuado sobre el motor por medio de salidas analógicas, conectadas a su vez a un puente $\mathrm{H}$, para colocar al motor en la posición indicada por el control PID. 


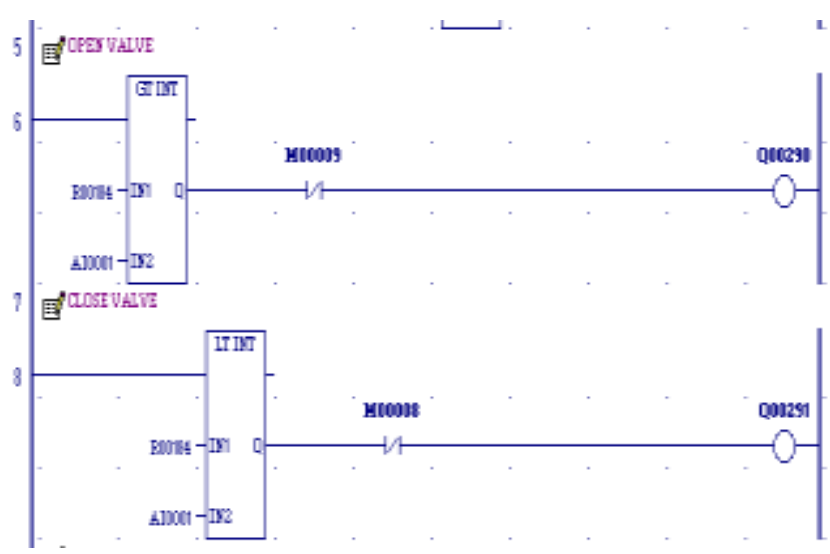

Figura 9. Algoritmo de control: Control del motor.

El motor debe posicionarse dentro de un rango de giro determinado (Figura 10), con la intención de no sobrepasar los límites de apertura y cierre de la válvula reguladora de gas, para evitar incidentes durante la operación del sistema.

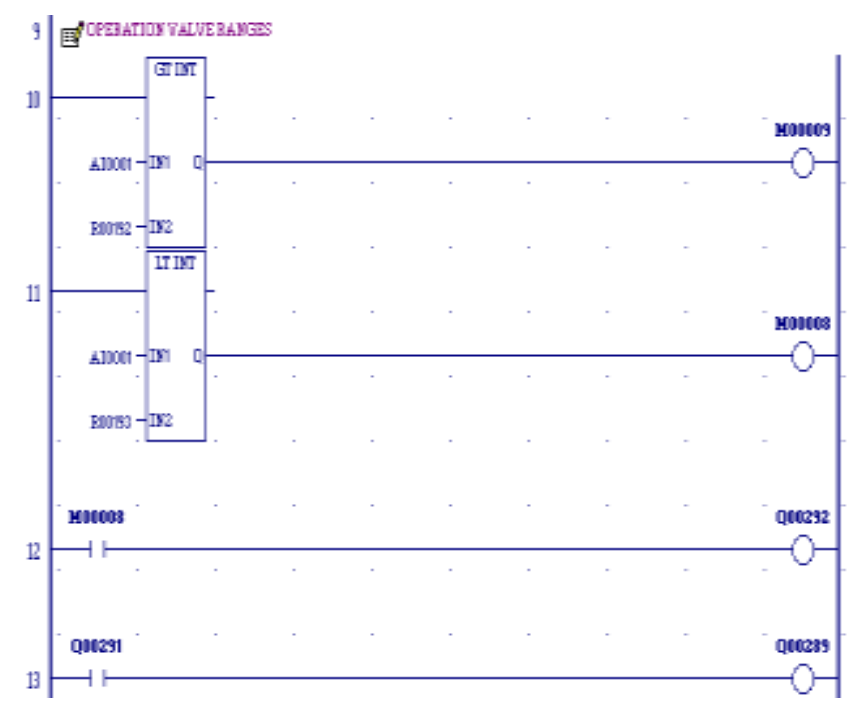

Figura 10. Algoritmo de control: Rango de operación del motor.

Como en todo proceso, es sumamente necesario implementar un sistema de seguridad que proteja la integridad del operador y del proceso. Para cubrir esta necesidad, el algoritmo de control permite la ejecución de un paro de emergencia, presentado en el diagrama de la Figura 11; de manera que el operador pueda detener el proceso, lo que implica cerrar totalmente la válvula de control, a través de una HMI (Human-Machine Interface; Interfaz Humano-Máquina).

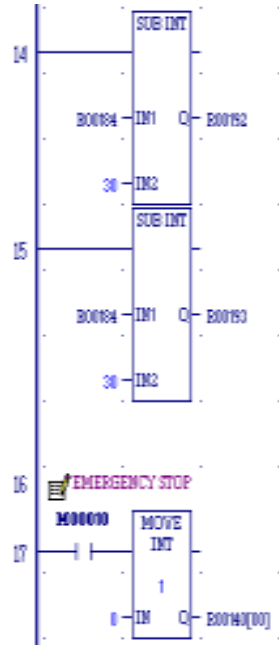

Figura 11. Algoritmo de control: Paro de emergencia.

La lógica de programación de los algoritmos de control fue desarrollada en lenguaje Diagrama Escalera (LD, Ladder Diagram) a través del software Proficy Machine Edition, e implementada en un PLC, específicamente en un PAC System RX3i de GE-Fanuc.

\section{Interfaz Humano-Maquina (HMI)}

La interfaz gráfica, desarrollada mediante el software Cimplicity Workbench, permite al operador ajustar el valor deseado de la temperatura y visualizar el valor que ésta tiene dentro del reactor en las etapas de maceración y cocción del proceso, así como poder realizar el control de la misma mediante la selección de alguno de los dos modos de operación, ya sea manual o automático. La Figura 12 muestra la pantalla principal de la HMI.

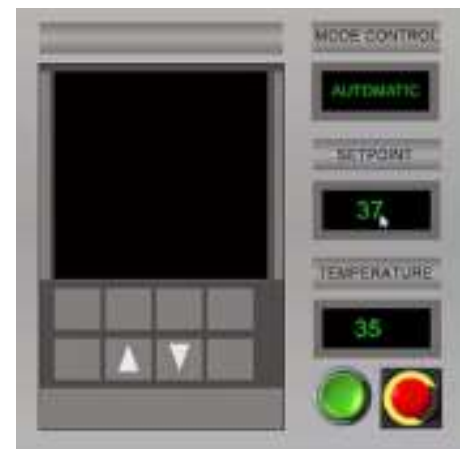

Figura 12. Pantalla principal de la HMI del operador.

Adicionalmente, como muestra la Figura 13, la HMI permite la opción de modificar los valores de las ganancias del controlador, con el propósito de poder realizar los ajustes necesarios para mejorar la sintonización del control PID; además de poder visualizar los estados de la temperatura y la posición de la válvula con respecto al tiempo. 


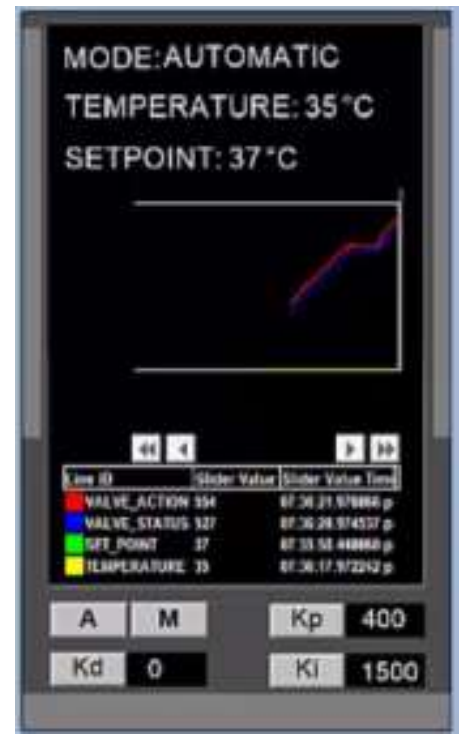

Figura 13. $\mathrm{HMI}$ - pantalla de ajustes del controlador.

\section{Conclusiones}

El presente trabajo constituye un proyecto académico terminal de aplicación práctica e implementación, con enfoque a la automatización de procesos productivos empleando tecnología de tipo industrial, PLC y $\mathrm{HMI}$ principalmente; como propuesta de alternativa de solución a la problemática que se presenta en el desarrollo de los procesos, en este caso la del control de temperatura en la maceración y cocción en la elaboración de cerveza artesanal. Esto ha permitido obtener resultados favorables en una pequeña empresa de ese giro, en una etapa de pruebas experimentales.

\section{Referencias}

[1] J. Huamán, «Gestiopolis» 2009. [En línea]. Available: http://www.gestiopolis.com/innovacionemprendimiento/mypes-en-el-peru.htm.

[2] D. Martínez, "Elaboración de cerveza". «Emagister» 2002. [En línea]. Available: http://www.emagister.com/uploads_courses/Comun idad_Emagister_48282_48281.pdf.

[3] R.S. Saurina, "Diseño de una micro-planta de fabricación de cerveza", 2015. [En línea]. Available: https://upcommons.upc.edu/discover?rpp=10\&etal= $0 \& q u e r y=$ cerveza\&group_by=none $\&$ page $=1$.

[4] P. Persson, "Towards autonomous PID control" Ph.D. dissertation, Department of Automatic Control, Lund Institute of Technology, 1992.

[5] H. Aboukheir, "Sistemas de Control II". Maracaibo. Universidad Rafael Belloso Chacín. Facultad de Ingeniería, 2006.
[6] M.E. Ramos. Investigación, 2015. Obtenido de UTN:

http://www.investigacion.frc.utn.edu .ar/sensores/te mperatura/termometro.com

[7] L. Cáliz, "Sistema de Control Predictivo para Separadores no Convencionales". Trabajo presentado como requisito para optar al grado de Magíster en Ingeniería de Control y Automatización de Procesos. Universidad Rafael Belloso Chacín, 2006.

[8] A.C. Solé, "Instrumentos industriales, su ajuste y calibración", Barcelona: Marcombo, 2009. 